\title{
SCREENING THE ONLY OPTION TO PREVENT CERVICAL CANCER
}

Cervical cancer takes many years to develop. Cervical changes can be detected before the development of cervical cancer. Screening helps to detect its early changes and hence prevention of cervical cancer is possible.

Cervical cancer is the second most common cancer in women globally. Approximately 500,000 cases are diagnosed annually and 250,000 deaths are recorded each year. $80 \%$ of the cases occur in developing countries where costly treatment modalities like surgery and radiotherapy are not accessible to most women. So, the primary approach is through prevention.

WHO has recently published a report on cervical cancer screening in developing countries in 2004 and recommended pap smear test as the screening technique which detects abnormal cervical cells. Cytological screening needs trained health personnel to take the cervical scrape, a reliable cytological laboratory, continuous access to high quality supplies and equipment, proven record system and effective referral mechanism for diagnosis and treatment. This is problematic in low resource setting. Thus, cytology is recommended as a screening strategy appropriate mainly in middle to high resource settings.

Visual approach to detect precursor cervical disease and cancer is explored and accepted by many developing countries. There are several types of visual screening. Early studies used unaided visual inspection, which involved simply performing a speculum examination to inspect the cervix for any signs of early cancer. This is known as down staging which is not sufficient to identify precursor lesions and cancer. Visual inspection with acetic acid (VIA) has been shown to be a more promising approach for identifying severe precursor lesion or cancer in women.
In VIA, the cervix is swabbed with 3-5\% acetic acid (vinegar) solution prior to visual examination. The changed characteristics of precursors proteins make abnormal cells temporarily white when exposed to the solution. A similar screening approach, visual inspection with lugol's iodine (VILI) also stains normal cervical cell brown, leaving abnormal cells with a yellow or unstained appearance.

Several studies have found VIA to be accurate but verification bias is a problem in most studies because a reference test is seldom performed on a study subjects.

There are a number of limitation that needs to be addressed before VIA can be recommended for routine use. The estimated specificity of VIA appear to be between $70 \%$ and $80 \%$. The positive predictive value of a VIA test ranges from $10 \%-30 \%$ when performed in an asymptomatic, non screened population.

Screening should ideally be targeted primarily at women between 35 and 50 years of age. These women are generally at high risk of developing precursor lesions or cervical cancer. A reasonable aim would be to screen every woman in the target group once in her life time at 40 years in countries with limited resources. It is well known that small proportion of low grade cervical lesion regress spontaneously or do not progress. Only $10-11 \%$ progress to high grade lesion or worsens within two years. About $1 / 3$ of high grade lesions will progress to invasive cancer within 10 years. This pattern has implications for cervical cancer prevention programme.

In Nepal, the untimely death of women due to cancer cervix can be minimized by adopting this simple, cost effective screening option of visual inspection with acetic acid. 